
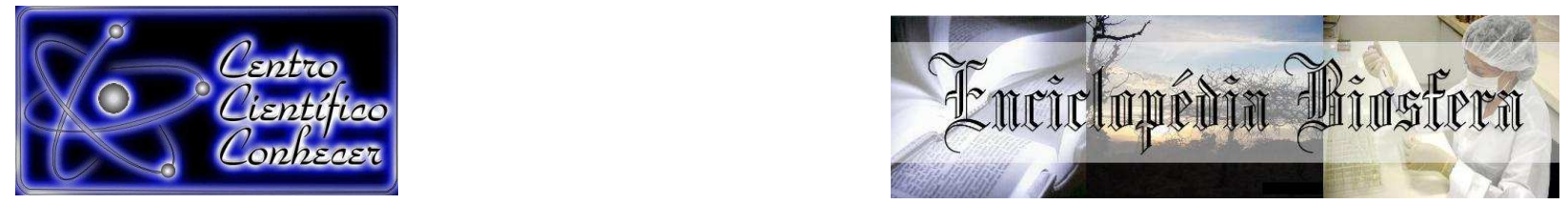

\title{
FUNÇÃO DA CENTRAL DE REGULAÇÃO DE LEITOS DO SISTEMA ÚNICO DE SAÚDE (SUS) NO ACESSO AO SERVIÇO DE INTERNAÇÃO HOSPITALAR NO MUNICÍPIO DE BELÉM, ESTADO DO PARÁ
}

Uêbem Fernandes Ramos ${ }^{1}$, Wilson Fernandes Ramos ${ }^{2}$

1 Graduado em Enfermagem pela Universidade do Estado do Pará - UEPA. E-mail: uebem@hotmail.com

2 Doutorando em Ciências Ambientais, Universidade Federal do Pará/UFPA, Belém, PA, Brasil.

Recebido em: 08/04/2017 - Aprovado em: 10/06/2017 - Publicado em: 20/06/2017 DOI: 10.18677/EnciBio_2017A129

\begin{abstract}
RESUMO
O Sistema Único de Saúde (SUS), desde a sua criação, expressa um verdadeiro avanço no Sistema de Saúde brasileiro e é pautado pelos princípios de universalidade, equidade e integralidade a partir das diretrizes organizacionais: descentralização e da participação popular. O presente estudo teve como objetivo descrever o funcionamento e operacionalização do serviço da central de regulação de leitos, bem como entender a percepção dos servidores (enfermeiros) sobre a central de regulação de leitos. Do ponto de vista metodológico, trata-se de um estudo de caso pautado em pesquisa descritiva e exploratória. As fontes utilizadas foram de natureza documental, incluindo elementos do próprio banco de dados do SISREG III, e primárias, obtidas por meio de entrevistas semi-estruturadas aplicadas junto aos enfermeiros. Os resultados apontam que 0 funcionamento e operacionalização da central de regulação de leitos é complexo, exigindo para a sua eficiência inúmeros órgãos e entidades privadas, que apresentam um papel fundamental para atendimento à saúde da população do Estado do Pará. Além disso, verificou-se que o sistema de regulação da Central de Leitos apresenta fragilidades, principalmente nas variáveis referentes à infraestrutura; ao relacionamento com as unidades solicitantes e executantes e à burocracia presente no processo do sistema de regulação.
\end{abstract}

PALAVRAS-CHAVE: Controles Formais da Sociedade, Enfermeiras e enfermeiros, Sistema Único de Saúde.

\section{FUNCTION OF THE HEALTH REGULATION CENTER OF THE SINGLE HEALTH SYSTEM (SUS) IN ACCESS TO THE HOSPITAL INTERVENTION SERVICE IN THE MUNICIPALITY OF BELÉM, STATE OF PARÁ}

\footnotetext{
ABSTRACT

The Health System, since its inception, expresses a real breakthrough in the Brazilian health system and is guided by the principles of universality, equity and integrity from the organizational guidelines: decentralization and popular ENCICLOPÉDIA BIOSFERA, Centro Científico Conhecer - Goiânia, v.14 n.25; p.1558 2017
} 
participation. This study aimed at describing the functioning and operation of the central service of beds regulation, as well as to understand the perception of servers (nurses) on the central regulation of beds. From a methodological point of view, it is a case study guided by descriptive and exploratory research. The sources used were documental nature, including elements of the SISREG III database itself, and primary, obtained through semi-structured interviews with nurses applied. The results show that the functioning and operation of the central regulation of beds is complex, requiring for their efficiency many agencies and private entities, which have a key role in health care in the State of Pará population. Furthermore, it was found that the regulatory system of the beds Center has weaknesses, particularly in the variables related to the infrastructure; the relationship with the requesting units and performers and bureaucracy present in the system of regulation process.

KEYWORDS: Social Control Formal, Unified Health System, Nurses

\section{INTRODUÇÃO}

A saúde no Brasil foi vista com maior importância a partir da Constituição Federal de 1988, onde é exposto que: "saúde é um direito de todos e dever do Estado" (BRASIL, 1988). Isso representou uma conquista para sociedade brasileira, a qual passou a ter acesso universal e pleno direito à assistência, bens e serviços de saúde, através do Sistema Único de Saúde (SUS).

Entretanto, essas conquistas estão longe de ser uma realidade, principalmente, em se tratando daqueles usuários que dependem exclusivamente do SUS, que são cerca $75 \%$ da população brasileira. Para tal, observam-se, diversas dificuldades e entraves no acesso aos serviços de saúde, demonstrando a necessidade de melhoria da qualidade, controle e regulação dos serviços ofertados pelo SUS (NARDI, 2015; COELI, et al., 2015). Essas dificuldades estão presentes em todas as regiões brasileiras, assim como, em capitais a exemplo de Belém (GOMES et al., 2015).

Nesse contexto, o Município de Belém, capital do Estado do Pará, representa uma das cidades mais importantes e populosas do país. Segundo o IBGE (2015) sua população é estimada em 1.439 .561 habitantes. Sua região metropolitana, chamada de grande Belém, que somam outros seis municípios, concentra aproximadamente $40 \%$ da riqueza e $35 \%$ da população do Estado. Isso demonstra a importância e influência dessa região para concentrar oferta de serviços e estabelecimentos de saúde do SUS (168 unidades) e a grande demanda da população pela assistência a saúde.

Diante da realidade socioeconômica do Município de Belém e do Estado do Pará, é de fundamental importância que existam o controle, o planejamento e a regulação do acesso aos bens e serviços de saúde por parte da população que necessita de atendimento pelo SUS. Para isso, faz-se necessário dispor de ferramentas e instrumentos de gestão e um sistema de saúde articulado, descentralizado e hierarquizado nos diversos níveis de complexidade e atenção à saúde (CONTREIRAS \& MATTA, 2015).

Em vista disso, no período de julho de 2013, a prefeitura de Belém, por meio de sua Secretaria Municipal de Saúde, implementou na central de leitos/internações de Belém, o software denominado, Sistema de Regulação (SISREG), que é uma ferramenta de gestão, controle e regulação do acesso dos usuários aos serviços de saúde do SUS (DA SILVA, 2016; CORDEIRO, 2016). Ademais, o mesmo auxilia na operacionalização e no fluxo de acesso dos usuários às internações em leitos 
hospitalares do SUS, ou seja, ordena a oferta e a demanda.

Este sistema vem sendo implantado em diversas unidades da federação ao longo dos anos e tem sido o grande responsável pela melhoria do acesso aos serviços de saúde através de marcações de consulta, exames, internações hospitalares, entre outras funcionalidades. Em particular, no Município de Belém, a implantação do SISREG, é considerada uma evolução, um passo importante para controle e regulação do acesso aos serviços de saúde pelos usuários do SUS (MARCON, et al., 2013).

Atualmente, a Central de Leitos do Município de Belém/PA recebe 8.780 pacientes cadastrados/mês, média de 293 pacientes cadastrados diariamente, provenientes de 209 unidades solicitantes, incluindo todos os demais 143 municípios do Estado do Pará, e até de outros estados da federação. Esse grande volume de pacientes cadastrados demonstra a importância e o papel do funcionamento da Central de Leitos de Belém, concomitantemente com o sistema (SISREG) como ferramenta de gestão e regulação em saúde.

Nesse sentido, estudos para entender as principais dificuldades no funcionamento e operacionalização da central de regulação, assim como do sistema de regulação, são essenciais para a proposição de soluções viáveis que contribuam para a geração de eficiência e eficácia na prestação desse serviço, e acesso da população às internações hospitalares pelo SUS. Dessa forma, objetivou-se descrever o funcionamento e operacionalização do serviço da central de regulação de leitos, bem como entender a percepção dos servidores (enfermeiros) sobre a central de regulação de leitos.

\section{MATERIAL E METODOS}

Trata-se de um estudo de caso, o qual tem por objetivo aprofundar a descrição de determinado fenômeno (YIN, 2015). Neste sentido, a pesquisa se classifica de acordo com a natureza como aplicada, com a finalidade de gerar conhecimentos para aplicação prática direcionada a solucionar problemas específicos. Quanto aos objetivos, classifica-se como descritiva, ao descrever as características dos fatos e fenômenos de determinada realidade. E exploratória, a qual visa proporcionar maior familiaridade com o problema com vistas a torná-lo explícito ou a construir hipóteses (BARDIN, 2011).

A coleta de dados sobre funcionamento e operacionalização da central de regulação de leitos, no Município de Belém/PA, foi do tipo pesquisa documental utilizando fontes documentais secundários, quais sejam ofícios, portarias e instruções normativas, além dos relatos de atores envolvidos neste processo. Para descrever o fluxo de atendimento e operacionalização do serviço da central de leitos, bem como os critérios de triagem e internação, usou-se o banco de dados do sistema nacional de regulação do SUS (SISREG III), o qual é utilizado pelo departamento de regulação da central de transferência e internação de leitos de urgência e emergência da secretaria de saúde do município de Belém do Pará.

Outra parte dos dados foi coletada através de entrevista, utilizando-se como instrumentos de pesquisa a entrevista semi-estruturada cujas perguntas foram previamente elaboradas e testadas ao longo da pesquisa. Esse instrumento de pesquisa conjuga perguntas abertas e fechadas, por meio das quais se busca coletar de informações dos sujeitos (PRODANOV \& FREITAS, 2013).

O instrumento foi constituído de sete questões/variáveis cujos itens utilizaram uma escala de cinco pontos do tipo Likert de respostas possíveis. A 
escala Likert requer que os entrevistados indiquem seu grau de insatisfação e satisfação com declarações relativas à atitude que está sendo medida (CALDEIRA, et al., 2014). A cada item foi atribuída uma escala qualitativa e outra quantitativa como segue: muito satisfeito (5), satisfeito (4), regular (3), insatisfeito (2) e muito insatisfeito (1). Essas entrevistas foram realizadas junto aos servidores da central de leitos de Belém/PA, que representam um universo de 16 enfermeiros, cuja função divide-se em regulador/autorizador e supervisor. E considerando que toda população de enfermeiros foi entrevistada, a presente pesquisa é classificada como censo. As variáveis consideradas na pesquisa foram: instalações físicas/equipamentos, recursos humanos, sistema de regulação (Sisreg III), relacionamento com as unidades solicitantes e executantes, burocracia, desajuste entre oferta e demanda e necessidade de utilização de protocolos de regulação.

Os dados foram sistematizados e analisados utilizando a estatística descritiva que "compreende o manejo dos dados para resumi-los ou descrevê-los, sem ir além, isto é, sem procurar inferir qualquer coisa que ultrapasse os próprios dados" (PRODANOV; FREITAS, 2013). O programa utilizado para fazer a análise estatística foi o aplicativo Excel do software Microsoft Office 2007, e os resultados foram expostos através de tabelas e gráficos.

\section{RESULTADOS E DISCUSSÕES \\ Funcionamento e operacionalização da central de regulação de leitos}

O Município de Belém, para melhor atender seus usuários, possui o Departamento de Regulação (DERE), denominado Complexo Regulador Municipal, formado pela Central de Leitos e Internação de Urgência (CLI), Central de Marcação de Consultas e exames (CMCE) e Central de Cirurgias Eletivas (CCE). Sua função consiste em controlar e regular o fluxo de acesso entre a oferta do serviço e a demanda dos usuários, nos diversos níveis de atenção e complexidade do sistema. A gestão desse complexo compete à Secretaria Municipal de Saúde de Belém (SESMA).

A Central de Regulação de Leitos e Internações atua, especificamente, nos serviços de alta complexidade (nível terciário de atenção), através da responsabilidade de regular o acesso aos leitos hospitalares dos estabelecimentos de saúde vinculados ao SUS. Esta surgiu como instrumentos de gestão que viabiliza o ordenamento da relação dos usuários com os prestadores de saúde e atua por fluxo de processo de regulação para o acesso aos leitos.

No acesso aos leitos hospitalares vinculados ao SUS, a Central de Regulação de Leitos e Internações atua por competência de atuação, onde cada ente que compõe o processo possui uma função delimitada. Assim, o fluxo do processo de regulação é composto pelas: unidades solicitantes, que correspondem a porta de entrada do sistema; a central de regulação, a qual se refere à ponte de ligação entre o paciente e o serviço de saúde; e por último a unidade executante que se relaciona no estabelecimento de saúde executando o serviço de internação e atendimento especializado ao usuário (FIGURA 1). 


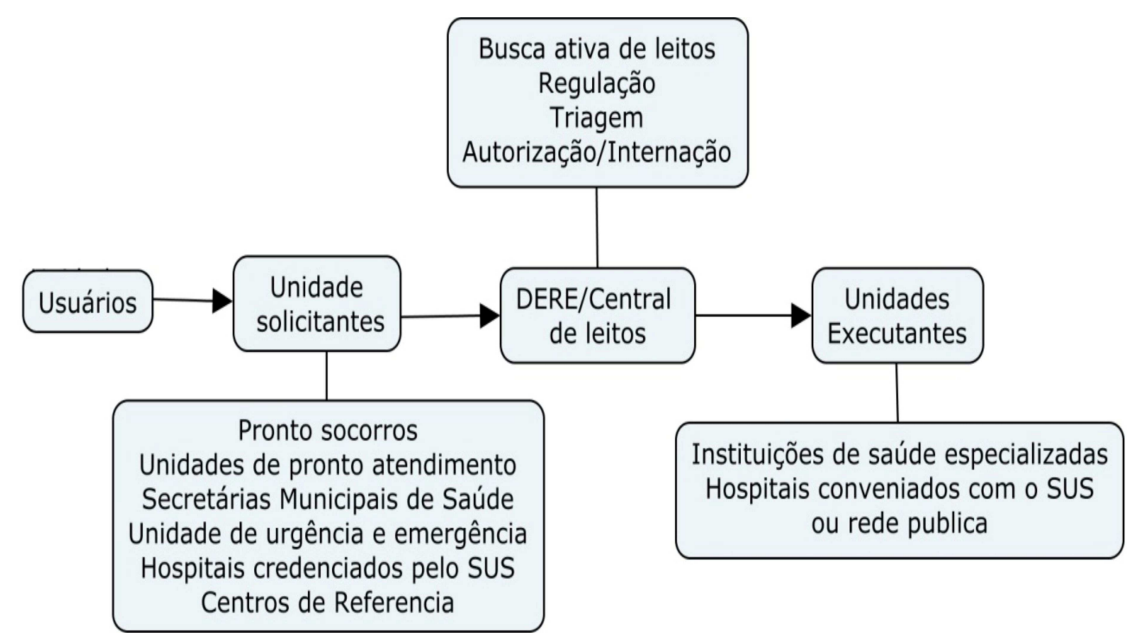

FIGURA 1 - Fluxo do processo de regulação de acesso a leitos.

Fonte: Elaborado pelos Autores

Tudo começa no processo de regulação, por meio do preenchimento de um laudo, chamado, Autorização de Internação Hospitalar $(\mathrm{AlH})$, pela unidade solicitante, quando o usuário ao necessitar de atendimento, internação ou procedimento de urgência dar entrada em uma unidade solicitante. Por conseguinte, o segundo passo é o cadastramento no Sistema online de Regulação (SISREG) em que ao fazer o cadastro é gerado um código, confirmando que o paciente entrará na fila virtual de espera por leito, na condição de cadastro pendente.

As unidades solicitantes correspondem a qualquer tipo de estabelecimento de saúde (unidades básicas, centros especializados, hospitais e secretarias municipais de saúde). Elas atuam, no papel de cadastrar o paciente no SISREG, solicitando transferência para internação em leitos hospitalares do SUS.

Nesse sentido, as unidades solicitantes do Município de Belém correspondem no total de 209 unidades. Desse total, 68\% correspondem às secretarias municipais de saúde do Estado do Pará (143); unidades da rede privada conveniada com o SUS (25); unidades da rede própria do Município de Belém (21); hospitais ou centros regionais (7); e secretarias municipais do Estado do Amapá (13).

Outro componente do processo de regulação corresponde a Central de Leitos de Belém a qual é o conjunto de infraestrutura, instalações, ação regulatória e recursos humanos. A central de leitos possui como função realizar a triagem, busca ativa de leitos vagos, regula, autoriza ou nega os cadastros solicitados no SISREG.

Por meio da ação regulatória é realizado o processo de autorização de Internação hospitalar, baseado nos protocolos clínicos e de regulação. No caso da Central de Leitos de Belém, a autorização dar-se-á através dos critérios de triagem e avaliação obedecida pelos servidores da Central de Leitos (Médicos ou Enfermeiros). Sendo assim, o paciente será internado em leito de urgência pelo SUS, de acordo com a disponibilidade de leito vago, perfil e quadro clínico do paciente e especialidade médica. 


\section{Perfil dos profissionais da central de regulação de leitos de Belém/Pa, com ênfase no profissional enfermeiro.}

A central de leitos presta um serviço essencial funcionando de maneira ininterrupta, 24 horas por dia, todos os dias da semana. Para isso, conta com um total de 28 agentes públicos organizados, através de escala de plantões e equipes de trabalho em número adequado e suficiente de servidores.

A equipe de trabalho da Central de Leitos de Belém é formada por um conjunto de servidores, multiprofissionais de diferentes níveis. No total, a equipe de servidores da central de leitos de Belém é formada por um coordenador (chefe do departamento), que atua propriamente como gestor; dois médicos que atuam cada nos horários matutino e vespertino, realizando a supervisão, triagem, avaliação e autorização para internação hospitalar; nove servidores de nível médio, atuando no setor administrativo; e os 16 enfermeiros, sendo 12 reguladores que realizam as triagens e regulação dos pacientes cadastrados e quatro enfermeiros supervisores, que fazem supervisão in loco nos hospitais executantes.

Analisando apenas o perfil do profissional enfermeiro, os resultados apontam que dos 16 servidores, $75 \%$ eram do sexo feminino, enfatizando a vocação feminina para a área da saúde. De acordo com descrição do perfil em gênero, a equipe de Central de Leitos segue a prerrogativa cultural da Enfermagem de que o "cuidar" está direcionado mais para o gênero feminino do que masculino (MOTTA, 2015; MACHADO, 2016). Enfatizado por RODRIGUES \& BARBIERI (2010) quando o predomínio de mulheres na enfermagem pode ser explicado pela capacidade de cuidar desenvolvida ancestralmente no convívio doméstico e não como qualidade inata e genética.

Em relação à faixa etária, verificou-se que a grande maioria está na faixa de 29 a 39 anos, seguido pela faixa de 40 a 50 anos e com menor representatividade está a faixa etária de 51 a 65 anos. Logo, pode-se inferir que os profissionais de enfermagem que atuam na Central de Leitos estão dentro da faixa que representa a idade madura em consonância com 0 processo de desenvolvimento e envelhecimento do homem (COSTA, 2016).

No que diz respeito à divisão de tarefas dos funcionários pesquisados, observou-se que $75 \%$ do quadro de profissionais desempenham o papel de regulador/autorizador, enquanto que $25 \%$ atuam como supervisores. Acredita-se que essa situação pode implicar em maior otimização dos serviços de triagem no que compete à $\mathrm{AlH}$, competência do regulador/autorizador. Por outro lado, o quantitativo de servidores enfermeiros, no total de 16, é suficiente para atender à demanda do serviço, uma vez que as atividades desempenhadas por esses profissionais consistem em uma análise burocrática de rotina de triagem e regulação dos pacientes. Dessa forma, o número de profissionais apresenta-se condizente com as atividades e a demanda para atendimento junto ao SISREG.

\section{Análise do grau de satisfação dos servidores enfermeiros da central de regulação, de acordo com as sete variáveis pesquisadas.}

Analisando-se a variável instalação físicas/equipamentos (Figura 2) nota-se que $50 \%$ dos enfermeiros entrevistados afirmam que, estão muito insatisfeitos; $37 \%$ encontram- se insatisfeitos; $13 \%$ consideram a infraestrutura física regular. Nenhum dos entrevistados mostrou-se satisfeito ou muito satisfeito. Demonstra-se com isso 
que a maioria dos profissionais se sente em geral, insatisfeita com a estrutura física e com equipamentos em uso no exercício de suas atividades profissionais.

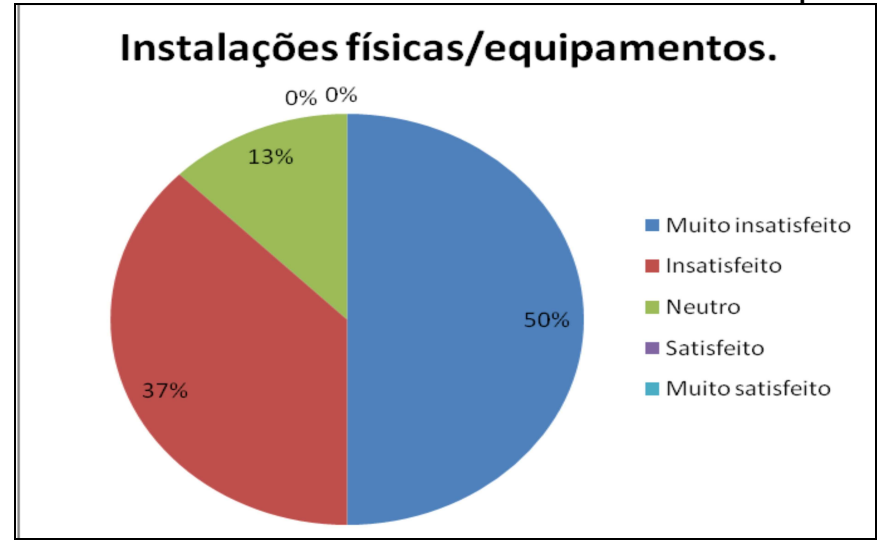

FIGURA 2 - Percentagem do grau de satisfação dos enfermeiros, de acordo com a variável Instalação físicas/equipamentos.

Fonte: Elaborado pelos Autores

Nesse sentido, ressalta-se que mediante 0 acentuado percentual de insatisfação em relação às instalações físicas e equipamentos, a pesquisa identifica esta variável como uma dificuldade ou talvez como obstáculo frente ao processo de regulação. Os recursos escassos impostos diariamente aos profissionais que trabalham com a regulação fomentam uma forte imagem de barreira e incluem no processo de trabalho a face dramática da regulação (GAWRYSZEWSKI et al., 2012). Diante desse quadro, há de se considerar como iniciativas que podem reverter tal cenário, a melhoria geral nessa variável como a substituição de computadores, sistema de telefonia e a velocidade de acesso à internet. Nesse sentido, a melhoria na infraestrutura física e nos equipamentos tendem a provocar mudanças positivas (ou negativas) no processo de trabalho (MARX, 1982; AMORIM, JUNIOR \& SHIMIZU, 2015).

Com relação a Recursos Humanos, observa-se que dentre os entrevistados $13 \%$ apresenta-se insatisfeito; $50 \%$ declaram-se regulares; $31 \%$ estão satisfeitos; e $6 \%$ muito satisfeito com o quantitativo de servidores (Figura 3 ).

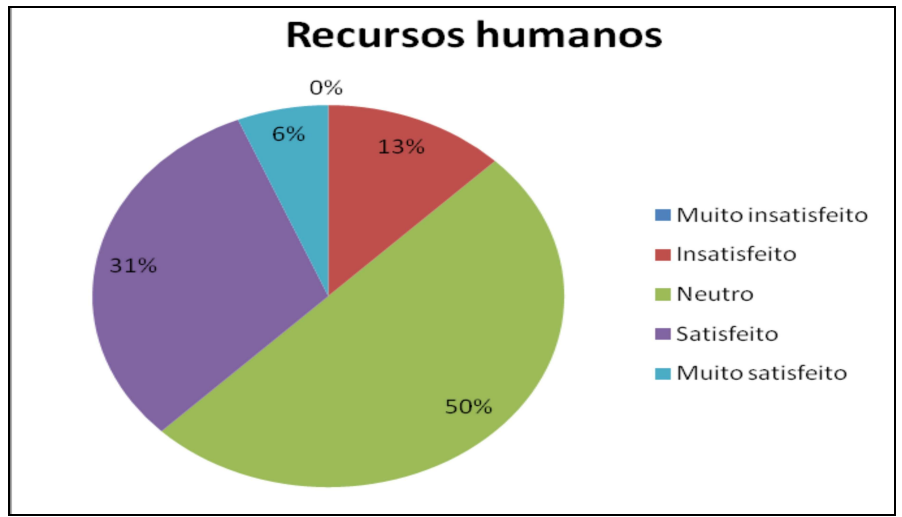

FIGURA 3 - Percentagem do grau de satisfação dos enfermeiros, de acordo com a variável recursos humanos.

Fonte: Elaborado pelos Autores 
Frente ao panorama traçado pelos resultados, nota-se a ausência de muito insatisfeitos em relação à variável em questão. Ressalta-se o elevado percentual dos entrevistados $(50 \%)$ que consideram o quantitativo de servidores regular, em relação ao grau de satisfação (31\%) e de insatisfação (13\%).

A análise da variável Sistema de Regulação (SISREG III) (Figura 10) demonstra que $27 \%$ estão insatisfeitos com o Sistema; $60 \%$ consideram seu funcionamento regular; $13 \%$ declaram satisfação com o Sistema. Frente aos dados, chama atenção o percentual mínimo de entrevistados (13\%) que se encontram satisfeitos com o SISREG (Figura 4).

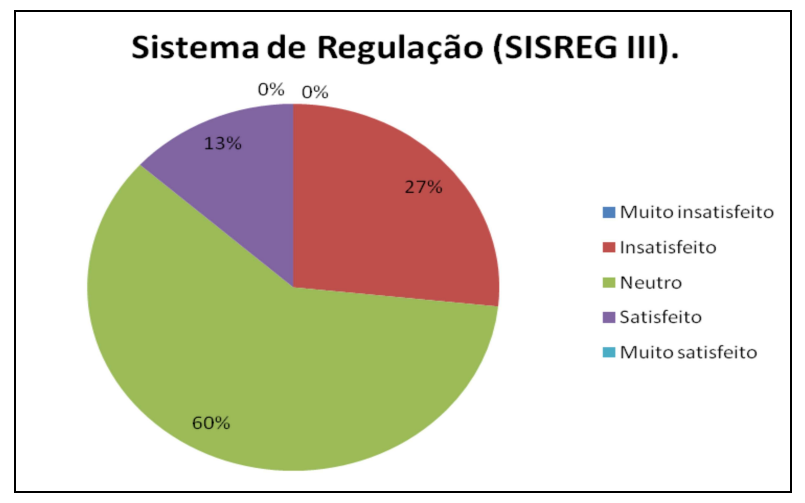

FIGURA 4: Percentagem do grau de satisfação dos enfermeiros, de acordo com SISREG III.

Fonte: Elaborado pelos Autores

Relacionando-se as duas variáveis (Grau de satisfação dos servidores com - SISREG e com as instalações físicas e equipamentos) é possível afirmar que o baixo grau de satisfação em relação ao SISREG está vinculado à insuficiência dos recursos estruturais disponíveis para o seu pleno serviço, haja vista que os dados demonstram que a dificuldade está mais na infraestrutura do que no próprio Sistema (Software do SISREG.)

Conforme LIEBER (2015), o que vai distinguir a formação de um sistema, ou não, é a organização, portanto, um sistema não é apenas uma coleção de entidades. Graças à organização, aquele agregado assume propriedades que não podem ser encontradas nas entidades isoladas. Dessa forma, compreende-se que as relações são estabelecidas entre si para que a atividade seja executada. Como no caso do SISREG, para que haja o acesso do paciente à Central de Leitos pressupõe que todas as etapas desse processo devem está interagindo de forma organizada.

Em relação à variável relacionamento com as unidades solicitantes e executantes, verifica-se que $37 \%$ dos entrevistados estão muito insatisfeitos quanto ao relacionamento com as unidades solicitantes e executantes; $25 \%$ encontram-se insatisfeitos; $25 \%$ mantiveram-se regulares; $13 \%$ declaram satisfação e não houve manifestação quanto ao item muito satisfeito (FIGURA 5). 


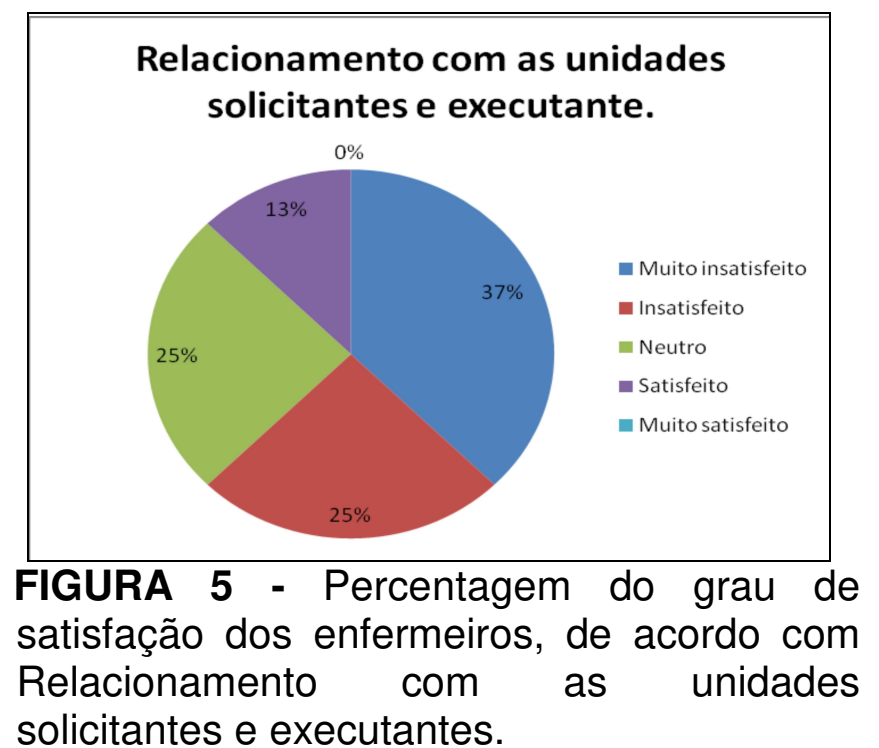

Fonte: Elaborado pelos Autores

A responsabilidade do enfermeiro está dividida em quatro aspectos: promover a saúde; prevenir a doença; estabelecer a saúde; e aliviar o sofrimento (COFEN, Resolução № 240/2000). Nesse sentido, o enfermeiro, ainda que de forma indireta na assistência ao paciente encontra-se no meio das informações divulgadas pelas unidades solicitantes e executantes. Assim, a falta de dialogo entre esses atores prejudica sobremaneira a população que necessita de um atendimento justo e eficiente (BADUY, 2011).

Infere-se, portanto, que o grau de insatisfação dos enfermeiros com relação às unidades prestadoras de serviço, está vinculado às falhas de comunicação tanto das unidades solicitantes, quanto das unidades executantes em relação a real situação do paciente em triagem, durante o processo de viabilização da internação pela Central de Leitos. Com relação à variável burocracia, $6 \%$ afirmam muita insatisfação; $50 \%$ insatisfação; $25 \%$ consideram regular; $19 \%$ satisfeitos; e não houve manifestação quanto item muito satisfeito (FIGURA 6).

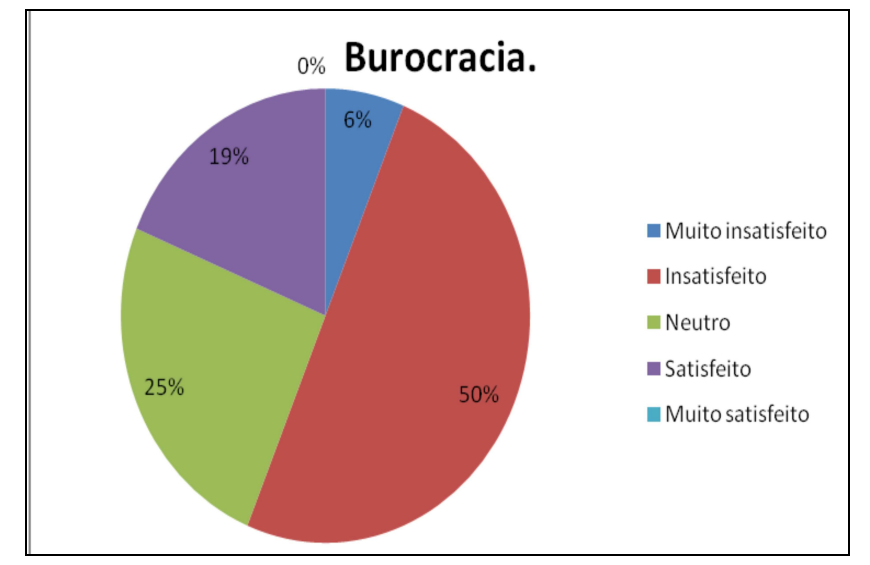

FIGURA 6 - Percentagem do grau de satisfação dos enfermeiros, de acordo com a variável burocracia.

Fonte: Elaborado pelos Autores 
Nesse contexto, os resultados apresentam alto grau de insatisfação dos servidores em relação à burocracia, constituindo-se como uma das dificuldades para a eficácia do trabalho, uma vez que o serviço da Central de Leitos exige comunicação formal dentro do SISREG, dos quais integra as unidades solicitantes e executantes a central de leitos. De acordo com Chiavenato, entende-se por burocracia:

\begin{abstract}
"Uma organização ligada por comunicações escritas. Regras, decisões e ações administrativas que são formuladas e registradas por escrito. Como as comunicações são feitas repetitiva e constantemente, a burocracia lança mão de rotinas e formulários para facilitar as comunicações e rotinizar 0 preenchimento de sua formalização" (CHIAVENATO, 2004, p.213).
\end{abstract}

Quanto as variáveis: relacionamento com as unidades solicitantes e executantes e burocracia, percebe-se, que dentre os entrevistados, a maioria manifesta insatisfação no que diz respeito ao relacionamento existente entre as unidades solicitantes e executantes, pressupondo-se relação com o nível de burocracia exercido dentro do trâmite processual do serviço, também designado como de insatisfação pelos profissionais.

A partir da análise dos dados, propõe-se que o gestor da Central de Leitos, em conjunto com os demais gestores das unidades solicitantes e executantes, deve estabelecer um protocolo local, em observância ao nacional, que apresente um conjunto de normas e rotinas que contenham os procedimentos de cadastro, triagem, regulação e internação do paciente, como forma de minimizar o índice de insatisfação dos servidores, e proporcionar melhorias na prestação do serviço.

Para tanto, sugere-se a identificação dos principais problemas do fluxo operacional e as falhas de comunicação internas ao sistema que pela falta de intempestividade das informações quanto ao paciente, dentro do SISREG, tornam o sistema na prestação de serviços, com retrabalho ou destinação inadequada dos leitos.

A análise da variável desajuste entre oferta e a demanda, demonstra que $69 \%$ dos entrevistados estão muito insatisfeitos; $25 \%$ encontram-se insatisfeitos; $6 \%$ consideram regular; e não houve manifestação quanto aos itens satisfeito e muito satisfeito (FIGURA 7).

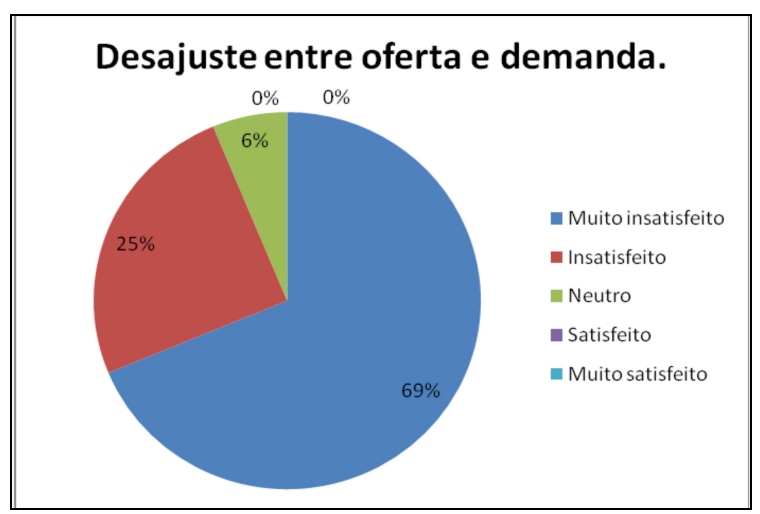

FIGURA 7 - Percentagem do grau de satisfação dos enfermeiros, de acordo com a variável desajuste entre oferta e demanda.

Fonte: Elaborado pelos Autores 
Há que se considerar que diante do quadro de insatisfação dos servidores quanto a essa variável, parte desse descontentamento está vinculado ao caráter de "impotência" em conseguir alocar o contingente de pacientes que diariamente necessitam de leitos. Por outro lado, Belém é um pólo receptor de pacientes de outros municípios, principalmente da Região Metropolitana de Belém e das regiões adjacentes à capital paraense, o que contribui para ampliar a demanda por leitos do município de Belém.

Sugere-se a ampliação da rede hospitalar, principalmente das especialidades com maior demanda e menor oferta de serviço, dentre as quais: UTI neurológica, UTI pediátrica e UTI oncológica. Ressalta-se ainda que no Estado do Pará, observa-se redução do número de leitos privados conveniados ao SUS, em oposição ao crescimento populacional e ao aumento da demanda por serviços de saúde. Porém essa ampliação depende da gestão das três de esferas de governo.

Por fim, na análise da variável necessidade de utilização de protocolos de regulação, $14 \%$ estão muito insatisfeitos; $36 \%$ insatisfeitos; $21 \%$ consideram regular; $29 \%$ declaram estarem satisfeitos e não houve manifestação quanto ao item muito satisfeito (FIGURA 8). Parte das dificuldades observadas reflete problemas mais amplos da gestão do trabalho em saúde no Brasil (MACHADO, 2016).

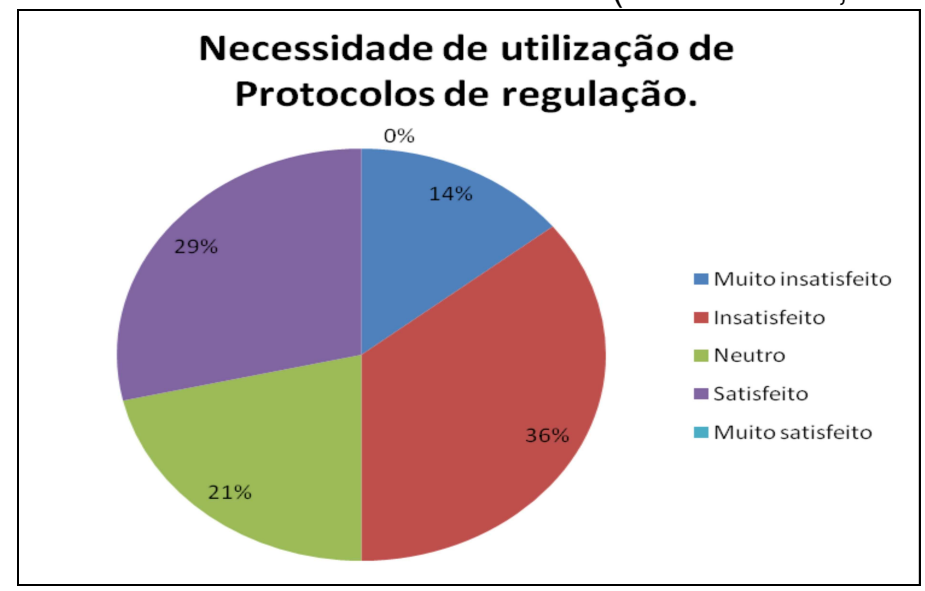

FIGURA 8: Percentagem do grau de satisfação dos enfermeiros, de acordo com a variável necessidade de utilização de Protocolos de regulação.

Fonte: Elaborado pelos Autores

Com relação ao uso do protocolo, percebe-se certa incoerência dentre os pesquisados que se encontram insatisfeitos, uma vez que os protocolos são ferramentas de trabalho que proporcionam melhor triagem da demanda para posterior encaminhamento do paciente. Destaca-se que a Central de Regulação de Leitos do Município de Belém segue o modelo nacional fornecido pelo Ministério da Saúde, não tendo um modelo próprio de protocolo. Nesse sentido, reforça-se a proposta apresentada anteriormente relacionada à variável Burocracia, quanto à necessidade de elaborar um Protocolo local para normatização dos procedimentos de cadastro, triagem, regulação e internação do paciente. 


\section{CONCLUSÃO}

O funcionamento e operacionalização da central de regulação de leitos são complexos, exigindo para a sua eficiência inúmeros órgãos e entidades privadas (hospitais privados), que apresentam um papel fundamental para a concretude do atendimento à saúde da população do Estado do Pará. O perfil da equipe da Central de Leitos, no que diz respeito aos enfermeiros, apresentou-se na faixa etária mais produtiva, sendo formada em sua maioria por mulheres.

A pesquisa ressaltou a insatisfação dos enfermeiros com a maioria das variáveis pesquisadas, mostrando que, a central de regulação de leitos deve investir em instalações físicas/equipamentos, diminuir burocracia, melhorar o relacionamento com as unidades solicitantes e executantes, bem como a adequação entre oferta e a demanda, oferecendo mais vagas no serviço de saúde.

Os resultados apresentados sinalizam para a necessidade de se rever os mecanismos de gestão uma vez que o percentual elevado de insatisfação em relação a variáveis ligadas diretamente ao processo burocrático, assim como na utilização dos próprios equipamentos de suporte da atividade, de modo geral, considerados como obsoletos.

O estudo apresenta como limitação a amostra bastante reduzida e a falta da analise da percepção de outros autores que estão envolvidos neste processo, além dos enfermeiros, o que pode ter enviesado a pesquisa.

\section{REFERÊNCIAS}

Constituição da República Federativa do Brasil de 1988. Disponível em: <http://www.planalto.gov.br/ccivil_03/constituicao/constituicao. htm>. Acesso em: 02 nov. 2015.

AMORIM, A.S.; JUNIOR, V.L.P.; SHIMIZU, H.E.; O desafio da gestão de equipamentos médico-hospitalares no Sistema Único de Saúde. Centro brasileiro de estudos de saúde (cebes), v. 39, n. 105, p. 350-362, 2015.

BADUY, R. S. et al. A regulação assistencial e a produção do cuidado: um arranjo potente para qualificar a atenção. Cadernos de Saúde Pública, Rio de Janeiro, v. 27, n. 2, p. 295-304, 2011.

BARBIERI, M., RODRIGUES, J., orgs. Memórias do cuidar: setenta anos da Escola da Escola Paulista de Enfermagem. Editora Unifesp: São Paulo, 2010. Disponível em: <http://books.scielo.org>. Acesso em: 16 nov. 2015.

BRASIL. Constituição (1988). Constituição da República Federativa do Brasil: promulgada em 5 de outubro de 1988. Organização do texto: Odete Medauar. 10. ed. São Paulo: RT, 2010.

CALDEIRA, S.; Metodologia de validação de Diagnósticos de Enfermagem: os modelos de Richard Fehring. 2014.

CHIAVENATO, I. Introduçao à teoria geral da administração: uma visão abrangente da moderna administração das organizações. $3^{a}$. ed. Rio de Janeiro: Elsevier, 2004.

COELI, C.M.; PINHEIRO, R.S.; CAMARGO JR, K.R.; Conquistas e desafios para o emprego das técnicas de record linkage na pesquisa e avaliação em saúde no Brasil. Epidemiologia e Serviços de Saúde, v. 24, n. 4, p. 795-802, 2015. 
CONTREIRAS, H.; MATTA, G.C.; Privatização da gestão do sistema municipal de saúde por meio de Organizações Sociais na cidade de São Paulo, Brasil: caracterização e análise da regulação. Cadernos de Saúde Pública, v. 31, n. 2, p. 285-297, 2015.

CORDEIRO, M.F.; SISREG: uma ferramenta de desafios e avanços para a garantia do direito a saúde. 2016.

COSTA, T.S.; Consultórios na rua do Distrito Federal e perfil dos profissionais de saúde no ano de 2015. 2016.

DA SILVA, D.A.J.; A Regulação no processo de trabalho do Núcleo de Apoio à Saúde da Família-resolutividade nas ações em saúde.Academus Revista Científica da Saúde, v. 1, n. 2, 2016.

GAWRYSZEWSKI, A.R.B.; OLIVEIRA, D.C.; GOMES, A.M.T.; Acesso ao SUS: representações e práticas de profissionais desenvolvidas nas Centrais de Regulação. Physis: Revista de Saúde Coletiva, v. 22, n. 1, 2012.

GOMES, K.C.; FERREIRA, V.R.; GOMES, D.L.; Qualidade da dieta de gestantes em uma unidade básica de saúde em Belém do Pará: um estudo piloto. Ciência \& Saúde, v. 8, n. 2, p. 54-58, 2015.

IBGE - Instituto Brasileiro de Geografia e Estatística [2015]. Disponível em: <http:// www.ibge.gov.br/_control01.html>. Acesso em: 02 dez. 2015.

LIEBER, R.R.; Teoria de Sistemas. Disponível em < http://www.inf.ufpr.br/urban /2015-2-Cl-220>. Acesso em 20 de nov.2015.

MACHADO, C.V.; Gestão do trabalho nas Unidades de Pronto Atendimento: estratégias governamentais e perfil dos profissionais de saúde.Cadernos de Saúde Pública, v. 32, n. 2, 2016.

MARX, K. Processo de trabalho e processo de produzir mais-valia. O capital. 8. ed. São Paulo: Difel, 1982.

MOTTA, L.J.;GONÇALVES, P.E.; LOPES, M.C.; Atenção Básica em Saúde Bucal: a experiência no curso de graduação. ABCS Health Sciences, v. 40, n. 3, 2015.

NARDI, A.C.F.et al. Resenha do livro Saúde Brasil 2014: uma análise da situação de saúde e das causas externas. 2015.

PRODANOV, C. C.; FREITAS, E. C. Metodologia do trabalho científico: métodos e técnicas da pesquisa e do trabalho acadêmico. 2. ed. Novo Hamburgo/RS: Universidade FEEVALE, 2013.

TRAVASSOS, C.; MARTINS, M. Uma revisão sobre os conceitos de acesso e utilização de serviços de saúde. Cadernos de Saúde Pública, Rio de Janeiro, v. 20, p. S190-S198, 2004. Suplemento 2.

VIANA, S. M. et al. Medindo as desigualdades em saúde no Brasil: uma proposta de monitoramento. Brasília: OPAS, IPEA, 2001.

YIN, R.K. Estudo de Caso-: Planejamento e Métodos. Bookman editora, 2015. 